\title{
Time-dependent kinetic energy metrics for Lagrangians of electromagnetic type
}

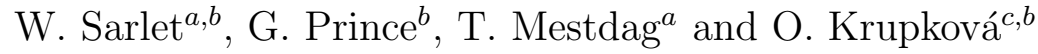 \\ ${ }^{a}$ Department of Mathematics, Ghent University \\ Krijgslaan 281, B-9000 Ghent, Belgium \\ ${ }^{b}$ Department of Mathematics and Statistics, La Trobe University \\ Bundoora, Victoria 3086, Australia \\ ${ }^{c}$ Department of Mathematics, The University of Ostrava \\ 30. dubna 22, 70103 Ostrava, Czech Republic
}

\begin{abstract}
We extend the results obtained in [12 about a class of Lagrangian systems which admit alternative kinetic energy metrics to second-order mechanical systems with explicit time-dependence. The main results are that a time-dependent alternative metric will have constant eigenvalues, and will give rise to a time-dependent coordinate transformation which partially decouples the system.
\end{abstract}

\section{Introduction}

In a recent contribution [12] two of us examined the following particular case of the inverse problem of Lagrangian mechanics: suppose that an autonomous second-order system admits a Lagrangian description such that the Hessian of the Lagrangian $L$ is the unit matrix; what are the conditions for the existence of an alternative kinetic energy metric for the same system, or expressed differently, an alternative multiplier for the inverse problem, which depends on the position variables only. Two observations we made are worth mentioning right away: (i) the starting assumption implies that the forces under consideration necessarily are of (generalized) electromagnetic type; (ii) if a second multiplier exists, it necessarily will have to be constant. We obtained several interesting results for such systems, one of them being that they necessarily decouple in coordinates which diagonalize the alternative metric.

It turns out to be quite interesting to enlarge the scope of applicability of the preceding study by allowing explicit time-dependence into the picture. This is the subject of the present paper. We will see indeed that such an extension leads to non-trivial complications, such as the fact that the determination of admissible scalar and vector potentials is no longer a separate issue. Nevertheless, the conclusion about partial decoupling of the system in appropriate coordinates will be shown to remain intact. 
The literature about the inverse problem of Lagrangian dynamics is vast. For a small sample of different techniques and mathematical tools which have been used, see [10, 2, 7, 6, 1, 3] and the list of references in the review paper [8]. A number of these techniques require quite advanced methods of differential geometry. Note that allowing explicit time-dependence in the framework is not always a straightforward matter; it involves, for example, passing from the vector bundle structure of a tangent bundle to the affine context of a first jet bundle. For specific aspects of time-dependence in this context, see for example [4, 9, 8, 13. The very concrete problem we are addressing here is much more computational in nature, so that it will be sufficient to rely on analytical expressions of the conditions for the existence of a Lagrangian, such as the ones developed in [10].

The second-order systems (SODEs for short) we start from are general non-autonomous differential equations in normal form, say

$$
\ddot{x}^{a}=F^{a}(t, x, \dot{x}), \quad a=1, \ldots, n .
$$

Geometrically they are the analytical expression of a second-order equation field $\Gamma$ living on the first jet bundle $J^{1} \pi$ of a bundle $\pi: E \rightarrow \mathbb{R}$, so

$$
\Gamma=\frac{\partial}{\partial t}+\dot{x}^{a} \frac{\partial}{\partial x^{a}}+F^{a}(t, x, \dot{x}) \frac{\partial}{\partial \dot{x}^{a}} .
$$

The local formulation of the general inverse problem is the question for existence of a (non-singular) multiplier matrix $g_{a b}(t, x, \dot{x})$, such that

$$
g_{a b}\left(\ddot{x}^{b}-F^{b}\right) \equiv \frac{d}{d t}\left(\frac{\partial L}{\partial \dot{x}^{a}}\right)-\frac{\partial L}{\partial x^{a}},
$$

for some Lagrangian function $L(t, x, \dot{x})$. The necessary and sufficient conditions for the existence of $L$ are generally referred to as the Helmholtz conditions, and when expressed in terms of conditions on the multiplier matrix, they read (see [10]):

$$
g_{a b}=g_{b a}, \quad \Gamma\left(g_{a b}\right)=g_{a c} \Gamma_{b}^{c}+g_{b c} \Gamma_{a}^{c}, \quad g_{a c} \Phi_{b}^{c}=g_{b c} \Phi_{a}^{c}, \quad \frac{\partial g_{a b}}{\partial \dot{x}^{c}}=\frac{\partial g_{a c}}{\partial \dot{x}^{b}}
$$

Here

$$
\Gamma_{b}^{a}:=-\frac{1}{2} \frac{\partial F^{a}}{\partial \dot{x}^{b}}
$$

geometrically represent the components of the canonical non-linear connection associated to $\Gamma$, so they are non-tensorial objects. On the other hand,

$$
\Phi_{b}^{a}:=-\frac{\partial F^{a}}{\partial x^{b}}-\Gamma_{b}^{c} \Gamma_{c}^{a}-\Gamma\left(\Gamma_{b}^{a}\right)
$$

are the components of a type $(1,1)$ tensor field along the projection $\pi_{0}^{1}: J^{1} \pi \rightarrow E$ called the Jacobi endomorphism. For a systematic development of the geometrical calculus along $\pi_{0}^{1}$, the interested reader can consult [13]. Further worth mentioning for later 
reference is that an integrability study of the conditions for the $g_{a b}$ leads to more algebraic restrictions, the first one of which, called the curvature condition, reads

$$
g_{a r} R_{b c}^{r}+g_{b r} R_{c a}^{r}+g_{c r} R_{a b}^{r}=0
$$

Here,

$$
R_{b c}^{a}:=H_{c}\left(\Gamma_{b}^{a}\right)-H_{b}\left(\Gamma_{c}^{a}\right), \quad \text { where } \quad H_{c}=\frac{\partial}{\partial x^{c}}-\Gamma_{c}^{r} \frac{\partial}{\partial \dot{x}^{r}}
$$

are components of the curvature tensor of the non-linear connection referred to before.

The concrete question we shall address in this paper is the characterization of (generally time-dependent) second-order systems which have a Lagrangian representation with a Euclidean kinetic energy term, and which admit at least one alternative Lagrangian representation for which the multiplier matrix does not depend on the velocities but may explicitly depend on time. The main conditions to be satisfied by such systems are developed in section 2; one of the conclusions is that the alternative multiplier $g$ can in fact be a function of time only. In section 3, it is shown that this $g$ will have constant eigenvalues and that a linear coordinate transformation which diagonalizes $g$ will decouple the system into lower dimensional Lagrangian systems. Explicit examples which illustrate the various aspects of the theory are presented in section 4 . In section 5 , we are led to an additional characterization of the systems under consideration, in the particular case that the connection coefficients are functions of time only.

\section{Lagrangians of electromagnetic type and alternative mul- tipliers}

Suppose $\Gamma$ is a SODE field on $J^{1} \pi$ with the property that, in some coordinate system, the unit matrix: $g_{a b}=\delta_{a b}$ is a multiplier matrix for the inverse problem. In fact, for simplicity, we shall take $\pi$ to be the trivial bundle $\pi: \mathbb{R} \times \mathbb{R}^{n} \rightarrow \mathbb{R}$. Since the unit matrix is the Hessian of the function $\frac{1}{2} \sum\left(\dot{x}^{a}\right)^{2}$, our assumption means that there exists a Lagrangian $L$ for the given SODE, which differs from that quadratic function at most by a linear function in the velocities, say $L=\frac{1}{2} \sum\left(\dot{x}^{a}\right)^{2}+A_{c}(t, x) \dot{x}^{c}-V(x, t)$. This implies that the 'forces' will be of the form $\left(A^{a}=\delta^{a b} A_{b}\right)$

$$
F^{a}=\left(\frac{\partial A^{b}}{\partial x^{a}}-\frac{\partial A^{a}}{\partial x^{b}}\right) \dot{x}^{b}-\frac{\partial V}{\partial x^{a}}-\frac{\partial A^{a}}{\partial t} .
$$

It further follows that the connection coefficients are skew-symmetric:

$$
\Gamma_{b}^{a}:=-\frac{1}{2} \frac{\partial F^{a}}{\partial \dot{x}^{b}}=\frac{1}{2}\left(\frac{\partial A^{a}}{\partial x^{b}}-\frac{\partial A^{b}}{\partial x^{a}}\right),
$$

while the components of the Jacobi endomorphism become

$$
\Phi_{c}^{a}=\frac{1}{2}\left(\frac{\partial^{2} A^{a}}{\partial x^{c} \partial x^{b}}+\frac{\partial^{2} A^{c}}{\partial x^{a} \partial x^{b}}-2 \frac{\partial^{2} A^{b}}{\partial x^{a} \partial x^{c}}\right) \dot{x}^{b}-\Gamma_{b}^{a} \Gamma_{c}^{b}+\frac{\partial^{2} V}{\partial x^{a} \partial x^{c}}+\frac{1}{2}\left(\frac{\partial^{2} A^{a}}{\partial t \partial x^{c}}+\frac{\partial^{2} A^{c}}{\partial t \partial x^{a}}\right)
$$


and are seen to be symmetric.

We want to investigate under what conditions there exists a second multiplier for such systems, which does not depend on the velocities. If $g$ does not depend on the velocities it follows from the requirement

$$
\Gamma\left(g_{a b}\right)=\frac{\partial g_{a b}}{\partial t}+\frac{\partial g_{a b}}{\partial x^{c}} \dot{x}^{c}=g_{a c} \Gamma_{b}^{c}+g_{b c} \Gamma_{a}^{c},
$$

that $g$ must actually be a function of $t$ only for which it must further hold that

$$
\dot{g}_{a b}(t)=g_{a c}(t) \Gamma_{b}^{c}+g_{b c}(t) \Gamma_{a}^{c}
$$

Notice that this implies

$$
g_{a c}(t) \frac{\partial \Gamma_{b}^{c}}{\partial x^{r}}+g_{b c}(t) \frac{\partial \Gamma_{a}^{c}}{\partial x^{r}}=0
$$

which is a somewhat weaker skew-symmetry property than in the autonomous case. The remaining Helmholtz condition $g_{a c} \Phi_{b}^{c}=g_{b c} \Phi_{a}^{c}$ is a linear expression in the $\dot{x}^{a}$ as well and thus also splits into two conditions. From the terms which are linear in the velocities it can be shown that the weaker condition (8) is sufficient to imply that (as in the autonomous case) we still must have

$$
\frac{\partial \gamma_{a r}}{\partial x^{b}}+\frac{\partial \gamma_{r b}}{\partial x^{a}}+\frac{\partial \gamma_{b a}}{\partial x^{r}}=0
$$

with $\gamma_{a b}:=g_{a c} \Gamma_{b}^{c}$. This may surprise at first sight, but it is fully consistent with the curvature condition (3) for this case. The remaining terms in the $\Phi$-condition, which in the autonomous case produced conditions on admissible scalar potentials $V$ only, now give rise to the following more complicated requirements, involving both the scalar and vector potential:

$$
\begin{aligned}
g_{a c}\left[\frac{\partial^{2} V}{\partial x^{c} \partial x^{b}}+\frac{1}{2}\left(\frac{\partial^{2} A^{c}}{\partial t \partial x^{b}}+\frac{\partial^{2} A^{b}}{\partial t \partial x^{c}}\right)-\Gamma_{r}^{c} \Gamma_{b}^{r}\right]= \\
g_{b c}\left[\frac{\partial^{2} V}{\partial x^{c} \partial x^{a}}+\frac{1}{2}\left(\frac{\partial^{2} A^{c}}{\partial t \partial x^{a}}+\frac{\partial^{2} A^{a}}{\partial t \partial x^{c}}\right)-\Gamma_{r}^{c} \Gamma_{a}^{r}\right] .
\end{aligned}
$$

Summarizing, we come to the following conclusion.

Proposition 1. Assume that the SODE $\Gamma$ on $\mathbb{R}^{2 n+1}$ admits a coordinate representation for which $\delta_{a b}$ is a multiplier matrix for the inverse problem of Lagrangian mechanics. Then the right-hand sides of the equations are of the form (4) for some functions $A^{a}$ and $V$. Any admitted alternative multiplier $g_{a b}$, not a multiple of the identity and independent of the velocities, must be a function of $t$ only, must satisfy the differential conditions (7), the curvature type conditions (9) with respect to the functions $A^{a}$, and the conditions (10) with respect to the functions $V$ and $A^{a}$. 
In the next section, we shall see that these conditions imply existence of a linear change of coordinates which partially decouples the given equations.

An interesting special case which complements the results of [12] is when the system with given forces (4) does depend explicitly on time, but we restrict the search for an alternative multiplier to a constant $g$. It then follows from (17) that the matrix $g \Gamma$ must be skew-symmetric. As a result the terms with two factors $\Gamma$ in the condition (10) cancel out, but compared to the autonomous case in [12] there remains a coupling between the scalar and vector potential. From the fact that also $g \partial \Gamma / \partial t$ will be skew-symmetric then, the remaining condition (10) can be further manipulated to reduce to

$$
g_{a c} \frac{\partial}{\partial x^{b}}\left(\frac{\partial V}{\partial x^{c}}+\frac{\partial A^{c}}{\partial t}\right)=g_{b c} \frac{\partial}{\partial x^{c}}\left(\frac{\partial V}{\partial x^{a}}+\frac{\partial A^{a}}{\partial t}\right) .
$$

\section{$3 \quad$ Diagonalization of $g$ and decoupling}

We observe that the differential conditions (7) for $g$ have the form of a Lax equation because the matrix $\Gamma_{b}^{a}$ is skew-symmetric. It follows that the eigenvalues of $g$ are constant. The point now is that the diagonalization of $g$ can be achieved by a linear coordinate change and that the connection coefficients, although not behaving tensorially under such operation, transform exactly in the way which is needed to contribute to decoupling of the system.

Let $P(t)$ be an orthogonal matrix which diagonalizes the symmetric matrix $g(t)$ and put $\bar{g}=P^{T} g P$, so that $\bar{g}$ is diagonal. Using an obvious matrix notation, the equation (7) can be written as

$$
\dot{g}=g \Gamma-\Gamma g .
$$

[It will always be clear from the context whether the notation $\Gamma$ refers to the soDE vector field or to the matrix of connection coefficients.] Multiplying on the left by $P^{T}$ and on the right by $P$, the equation transforms to

$$
\dot{\bar{g}}=\bar{g}\left(\bar{\Gamma}+P^{T} \dot{P}\right)-\left(\bar{\Gamma}+P^{T} \dot{P}\right) \bar{g},
$$

where $\bar{\Gamma}=P^{T} \Gamma P$. Notice that $\bar{\Gamma}$ is still skew-symmetric and the same is true for $\widetilde{\Gamma}:=\bar{\Gamma}+P^{T} \dot{P}$. It trivially follows from the fact that $\bar{g}$ is constant that the commutator in the right-hand side is zero.

Now consider the linear, time-dependent coordinate transformation $x=P(t) y$ with inverse $y=P(t)^{T} x$. Both $g_{a b}$ and $\Phi_{b}^{a}$ are actually components of tensor fields along $\pi_{0}^{1}: J^{1} \pi \rightarrow E$ of the form (see [13])

$$
g=g_{a b} \theta^{a} \otimes \theta^{b}, \quad \Phi=\Phi_{b}^{a} \theta^{b} \otimes \frac{\partial}{\partial x^{a}} \quad \text { with } \quad \theta^{a}=d x^{a}-\dot{x}^{a} d t .
$$

Hence they behave well under time-dependent coordinate transformations, meaning that their components in the new variables under consideration will be $\bar{g}$ and $\bar{\Phi}=P^{T} \Phi P$. 
Less obvious is what happens to the non-tensorial matrix of connection coefficients. It is a straightforward computation, however, to verify that the transformed second-order differential equations for the $y$-variables contain the following linear part in the velocities:

$$
\ddot{y}=-2\left(P^{T} \Gamma P-\dot{P}^{T} P\right) \dot{y}+\ldots
$$

Thus, the connection coefficients $\widetilde{\Gamma}_{b}^{a}$ of the transformed equations are precisely the components of the matrix $\widetilde{\Gamma}$ introduced above. Since $\widetilde{\Gamma}$ commutes with the diagonal matrix $\bar{g}$, it has a block diagonal structure, the dimension of each block being determined by the multiplicity of an eigenvalue of $g$. It follows that, if $A_{\alpha}$ and $B_{\beta}$ refer to coordinates of different blocks, the transformed forces $\widetilde{F}$ will have the property $\partial \widetilde{F}^{A_{\alpha}} / \partial \dot{y}^{B_{\beta}}=0$. But we also have $\bar{g} \bar{\Phi}=\bar{\Phi} \bar{g}$, meaning that $\bar{\Phi}$ has the same block diagonal structure, and from the coordinate expression of $\bar{\Phi}_{b}^{a}$ (see (즈), but to be read in the new variables), it then further follows that also $\partial \widetilde{F}^{A_{\alpha}} / \partial y^{B_{\beta}}=0$. Hence, the transformed equations decouple into independent subsystems, one for each eigenspace of $g$.

Proposition 2. Assume that a given SODE $\Gamma$ satisfies the conditions of proposition 1. Then the linear time-dependent coordinate transformation which diagonalizes the second multiplier $g_{a b}(t)$ will decouple the system into a number of separate SODEs, one for each distinct eigenvalue of $g$, and these eigenvalues are constants.

As we explained in 12 such a decoupling property was already established, for autonomous systems, in work of Ferrario et al [5], but to the best of our knowledge, it was not observed before that allowing time-dependence in the picture does not destroy it. Observe also that the transformed system (and hence every independent subsystem) still has the property of admitting the unit matrix as multiplier, because $P^{T} I_{n} P=I_{n}$. As a result, a reverse engineering technique to construct all systems under consideration goes as follows: start from a number of say $k$ independent SODEs with forces of type (4); multiply each of them with a different constant factor $\lambda_{k}$, thus creating a diagonal multiplier $\bar{g}$ for the union of the $k$ systems; then apply an arbitrary (possibly time-dependent) linear orthogonal transformation to the overall system; the resulting coupled system will be of the type characterized in Proposition 1. Note that each choice of a single equation at the start of this process, corresponding to an eigenvalue with multiplicity one of the $g$ under construction, will only contain a scalar potential. Since the group of orthogonal transformations is finitely generated, one can imagine that such a reverse engineering technique may be helpful in an attempt to classify all systems in the category under consideration. But there would of course, in such a classification, be room for an unlimited number of ways of putting together decoupled systems in the first place.

The more interesting approach for identifying suitable examples, therefore, is to start from an arbitrary SODE with forces (4) and to try to solve the conditions of Proposition 1 for the identification of admissible scalar and vector potentials and the construction of an associated alternative (possibly time-dependent) kinetic energy function. This is the line of approach we will adopt as much as possible in the next section. 


\section{Examples}

Consider a system $\ddot{x}^{a}=F^{a}(x, \dot{x}, t)$, where the $F^{a}$ are of the form (44). In the autonomous case we were able to obtain relevant information about the form of admissible vector potentials and the alternative multiplier $g$ by imposing first that the matrix $\gamma:=g \Gamma$ had to be skew and satisfy the curvature type condition (9). The remaining Helmholtz condition $g \Phi=(g \Phi)^{T}$ then resulted essentially in equations for the admissible scalar potential $V$. The situation is more complicated in the time-dependent case now, for the following three reasons: firstly, $\gamma$ need not be skew, instead, in view of (8) , we have the weaker condition that $\gamma-\gamma^{T}$ must be a function of time only; secondly, the information about $g$ which can be gathered in the process of analysing $\gamma$ will be far less conclusive, because $g$ still has to satisfy the differential equation (7) in the end; thirdly, the remaining algebraic condition (10) will in general not reduce to a separate issue concerning admissible functions $V$. As we shall show below, however, we can still get a long way in constructing examples for $n=2$ and $n=3$ by staying close to the procedure we followed in the autonomous case.

Taking $n=2$, and denoting the elements of the multiplier $g$ for shorthand by $a_{1}=$ $g_{11}, a_{2}=g_{22}, b=g_{12}=g_{21}$, the fact that $\gamma-\gamma^{T}$ can only depend on $t$ implies that $b \Gamma_{2}^{1}$ and $\left(a_{1}-a_{2}\right) \Gamma_{2}^{1}$ must be functions of time only. It follows that $\Gamma_{2}^{1}$ cannot depend on the $x$-coordinates either (which we write with lower indices here); putting $\Gamma_{2}^{1}=\sigma(t)$, the vector potential must satisfy

$$
\frac{\partial A_{1}}{\partial x_{2}}-\frac{\partial A_{2}}{\partial x_{1}}=2 \sigma(t)
$$

and is determined, up to a gradient, by

$$
A_{1}(t, x)=\sigma(t) x_{2}, \quad A_{2}(t, x)=-\sigma(t) x_{1} .
$$

The freedom of adding a gradient is irrelevant here since this adds to the Lagrangian $L$ we started from merely a total-time derivative term, plus a term which can be absorbed into the as yet undetermined scalar potential $V$. Expressed differently, we can assume without loss of generality that in dimension $n=2$ the system we start from can be written in the form

$$
\begin{aligned}
& \ddot{x}_{1}=-2 \sigma(t) \dot{x}_{2}-\dot{\sigma} x_{2}-\frac{\partial V}{\partial x_{1}}, \\
& \ddot{x}_{2}=2 \sigma(t) \dot{x}_{1}+\dot{\sigma} x_{1}-\frac{\partial V}{\partial x_{2}} .
\end{aligned}
$$

Quite remarkably, if we now move to the remaining algebraic condition (10), all terms not involving $V$ cancel out and we end up with the following condition for admissible functions $V$ :

$$
b\left(\frac{\partial^{2} V}{\partial x_{2}^{2}}-\frac{\partial^{2} V}{\partial x_{1}^{2}}\right)=\left(a_{2}-a_{1}\right) \frac{\partial^{2} V}{\partial x_{1} \partial x_{2}} .
$$


By way of example we proceed to determine admissible (time-dependent) quadratic potentials. Putting

$$
V=\frac{1}{2} k(t) x_{1}^{2}+l(t) x_{1} x_{2}+\frac{1}{2} m(t) x_{2}^{2}
$$

the condition becomes

$$
b(m-k)=\left(a_{2}-a_{1}\right) l
$$

and we will only discuss the generic case here that $l \neq 0$ and $m \neq k$.

Putting $l(t)=(m(t)-k(t)) \rho(t)$ the multiplier must have the form

$$
g=\left(\begin{array}{cc}
a_{1} & \left(a_{2}-a_{1}\right) \rho \\
\left(a_{2}-a_{1}\right) \rho & a_{2}
\end{array}\right)
$$

and the corresponding admissible potentials read

$$
V=\frac{1}{2} k x_{1}^{2}+(m-k) \rho x_{1} x_{2}+\frac{1}{2} m x_{2}^{2} .
$$

But now $g$ still must satisfy the differential equations (7). Putting $a_{2}=C-a_{1}$, where $C$ represents the constant trace of $g$, these equations reduce to:

$$
\begin{aligned}
\dot{a}_{1} & =2\left(2 a_{1}-C\right) \rho \sigma \\
\dot{\rho} & =-\left(1+4 \rho^{2}\right) \sigma .
\end{aligned}
$$

The case $2 a_{1}-C=0$ has to be excluded since $g$ must be kept different from a multiple of the identity. It is convenient to introduce a new time-scale $\tau:=\int \sigma(t) d t$; the equations then are easy to integrate, giving

$$
\rho(\tau)=\frac{1}{2} \tan (-2 \tau+A), \quad 2 a_{1}(\tau)-C=B \cos (-2 \tau+A),
$$

where $A$ and $B$ are constants.

In conclusion, with $k(t), l(t), \sigma(t)$ arbitrary functions of time, $\alpha(t)=-2 \int^{t} \sigma(s) d s+A$ and $\rho(t)=\frac{1}{2} \tan \alpha$, the admissible scalar and vector potentials are given by (14) and (12) respectively, and the alternative multiplier is

$$
g=\left(\begin{array}{cc}
\frac{1}{2}(C+B \cos \alpha) & -\frac{1}{2} B \sin \alpha \\
-\frac{1}{2} B \sin \alpha & \frac{1}{2}(C-B \cos \alpha)
\end{array}\right) .
$$

The eigenvalues of $g$ are $\lambda_{1}=\frac{1}{2}(C-B), \lambda_{2}=\frac{1}{2}(C+B)$ and corresponding eigenvectors read $(\sin \alpha, \cos \alpha+1),(\sin \alpha, \cos \alpha-1)$. They have to be scaled to norm 1 to produce the columns of an orthogonal matrix which diagonalizes $g$. For testing the statement about decoupling in Proposition 2, however, one can relax the requirement that $P$ be orthogonal. Indeed, if we look at the related coordinate transformation in the form $y=P^{T} x$ whereby the eigenvectors now appear as rows, it is clear that an overall factor in the defining relation of the $y$-variables cannot be an obstruction to decoupling. One can verify that the transformation

$$
\begin{aligned}
& y_{1}=\sin \alpha x_{1}+(\cos \alpha+1) x_{2} \\
& y_{2}=\sin \alpha x_{1}+(\cos \alpha-1) x_{2},
\end{aligned}
$$


effectively takes care of that.

Let's move now to systems with three degrees of freedom, for which the problem is more intricate because the curvature condition (9) comes into play. To better illustrate the effect of allowing time-dependence we try to stay close here to the analysis and notations of the $n=3$ example in [12. As indicated before, the matrix $\gamma=g \Gamma$ need not be skewsymmetric now, but its symmetric part, according to (8) can depend on time only. In particular, from the diagonal elements of this symmetric part, the following expressions (which had to be zero in the autonomous case) now must be certain functions of time (which we will not specify at the moment):

$$
g_{12} \Gamma_{2}^{1}+g_{13} \Gamma_{3}^{1}, \quad g_{12} \Gamma_{2}^{1}-g_{23} \Gamma_{3}^{2}, \quad g_{13} \Gamma_{3}^{1}+g_{23} \Gamma_{3}^{2},
$$

and we can view this as a linear system for the $\Gamma_{b}^{a}(t, x)$ (with time-dependent coefficients) which has zero determinant. Except for special cases where the rank of the coefficient matrix is lower than two (and which we will not discuss), solving this algebraically for the $\Gamma_{b}^{a}$ will give rise to relations of the type

$$
\Gamma_{3}^{1}=a(t) \Gamma_{2}^{1}+\tilde{a}(t), \quad \Gamma_{3}^{2}=b(t) \Gamma_{2}^{1}+\tilde{b}(t),
$$

for certain functions $a, b, \tilde{a}, \tilde{b}$. The sort of privileged role which $\Gamma_{2}^{1}(t, x)$ plays in these expressions is just a matter of renumbering the variables, if necessary. Substituting this structural information back into the linear equations from which it was obtained, we see that the product of $\Gamma_{2}^{1}(t, x)$ with any of the factors

$$
g_{12}+a g_{13}, \quad g_{12}-b g_{23}, \quad a g_{13}+b g_{23},
$$

must be some function of time only. There are then two cases to be considered: either $\Gamma_{2}^{1}$ also depends on time only, and then the same is true for $\Gamma_{3}^{1}$ and $\Gamma_{3}^{2}$; or we insist that $\Gamma_{2}^{1}$ should depend on at least one of the $x$-coordinates, and then the above mentioned factors necessarily must be zero. We investigate the second option first.

With $\Gamma_{3}^{1}$ and $\Gamma_{3}^{2}$ as specified above, the off diagonal elements of the symmetric part of $\gamma$ give us three more algebraic conditions, which are affine linear in $\Gamma_{2}^{1}$. Since we assume $x$-dependence in $\Gamma_{2}^{1}$, such relations can hold true only if the coefficients of $\Gamma_{2}^{1}$ vanish which yields

$$
g_{11}-g_{22}=b g_{13}+a g_{23}, \quad a\left(g_{11}-g_{33}\right)=g_{23}-b g_{12}, \quad b\left(g_{22}-g_{33}\right)=-a g_{12}-g_{13} .
$$

We now have sufficient elements to get an idea of the possible structure of the multiplier $g$. We must have $g_{12}=-a g_{13}=b g_{23}$ and to make $a g_{13}+b g_{23}$ zero as well, we can put $g_{13}=-\rho b, g_{23}=\rho a$, which implies that $g_{12}=\rho a b$. Putting further $g_{11}=\rho c$ (which defines the function $c(t)$ ), the last three conditions fix $g_{22}$ and $g_{33}$. The result is that the multiplier $g$ will have the structure:

$$
g=\rho\left(\begin{array}{ccc}
c & a b & -b \\
a b & c+b^{2}-a^{2} & a \\
-b & a & c+b^{2}-1
\end{array}\right),
$$


where $a, b, c$ and $\rho$ are all functions of time which will have to be determined from the differential equations which $g$ must satisfy. It is instructive at this stage to compare with the situation in the autonomous case (see [12]). It is not true here that if $g$ is a multiplier matrix, then $\rho(t) g$ is one too, so that the overall factor cannot be disregarded right away.

In dimension three the curvature type condition contains one requirement and with the information gathered so far, it can be seen to reduce to

$$
\frac{\partial \Gamma_{2}^{1}}{\partial x_{3}}-a \frac{\partial \Gamma_{2}^{1}}{\partial x_{2}}+b \frac{\partial \Gamma_{2}^{1}}{\partial x_{1}}=0
$$

which is formally the same as in the autonomous case. The fact that the coefficients depend on time now is not an obstruction to take over suitably adapted results we know from the autonomous situation. The general solution of the above linear partial differential equation is an arbitrary function $\Gamma_{2}^{1}=f(t, u, v)$ where

$$
u(t, x)=x_{1}-b(t) x_{3}, \quad v(t, x)=x_{2}+a(t) x_{3} .
$$

This means that admissible vector potentials must satisfy

$$
\begin{aligned}
& \frac{\partial A_{1}}{\partial x_{2}}-\frac{\partial A_{2}}{\partial x_{1}}=2 f(t, u, v) \\
& \frac{\partial A_{1}}{\partial x_{3}}-\frac{\partial A_{3}}{\partial x_{1}}=2 a(t) f(t, u, v)+2 \tilde{a}(t) \\
& \frac{\partial A_{2}}{\partial x_{3}}-\frac{\partial A_{3}}{\partial x_{2}}=2 b(t) f(t, u, v)+2 \tilde{b}(t) .
\end{aligned}
$$

The right-hand sides are the components of a closed 2 -form indeed (with $t$ treated as parameter), and a particular solution for the $A_{i}$ then is given by

$$
\begin{aligned}
& A_{1}=2 v \int_{0}^{1} s f(t, s u, s v) d s+\tilde{a}(t) x_{3}, \\
& A_{2}=-2 u \int_{0}^{1} s f(t, s u, s v) d s+\tilde{b}(t) x_{3}, \\
& A_{3}=-2(a u+b v) \int_{0}^{1} s f(t, s u, s v) d s-\tilde{a}(t) x_{1}-\tilde{b}(t) x_{2} .
\end{aligned}
$$

The general solution differs from this particular one by a gradient, but as argued in the preceding example, this gradient term can be incorporated into the as yet undetermined scalar potential, up to a total time derivative. We have obtained valuable information about the structure of $g$ and the vector potential now, but it is clear that the freedom in selecting functions $a, b, c$ and $\rho$ will be severely restricted if we now go back to the 
differential conditions (7). They read:

$$
\begin{aligned}
& \frac{d}{d t}(\rho c)=2 \rho \tilde{a} b, \\
& \frac{d}{d t}\left(\rho\left(c+b^{2}-a^{2}\right)\right)=-2 \rho \tilde{b} a, \\
& \frac{d}{d t}\left(\rho\left(c+b^{2}\right)\right)=2 \rho(\tilde{b} a-\tilde{a} b), \\
& \frac{d}{d t}(\rho a b)=\rho(b \tilde{b}-a \tilde{a}), \\
& \frac{d}{d t}(\rho a)=\rho\left(\tilde{b}\left(1-a^{2}\right)+\tilde{a} a b\right), \\
& \frac{d}{d t}(-\rho b)=\rho\left(\tilde{a}\left(1-b^{2}\right)+\tilde{b} a b\right) .
\end{aligned}
$$

It follows from the second and third equation that we must have

$$
\frac{d}{d t}\left(\rho a^{2}\right)=2 \rho(-\tilde{a} b+2 \tilde{b} a)
$$

Combining this with information coming from the fifth and sixth equation, one can derive the condition

$$
a \tilde{b}-b \tilde{a}=0
$$

and subsequently that

$$
\dot{a}=\tilde{b} \quad \text { and } \quad \dot{b}=-\tilde{a},
$$

which in turn implies that $a^{2}+b^{2}$ is constant, and from the third equation also that $\rho\left(c+b^{2}\right)$ is constant. Finally, going back to the fifth and sixth equation, which reduce to

$$
\frac{d}{d t}(\rho a)=\rho \tilde{b}, \quad \frac{d}{d t}(\rho b)=-\rho \tilde{a}
$$

and using the equations for $a$ and $b$ just obtained, it follows that actually also $\rho$ must be constant and can therefore, from now on, without loss of generality be put equal to 1 . With these restrictions, all six equations about $\dot{g}$ are now satisfied. We conclude that the freedom in constructing alternative multipliers consists of two arbitrary constants and one arbitrary function of time. Take $a(t)$ to be arbitrary for example, and choose two constants $c_{1}$ and $c_{2}$, then define $b$ by $a^{2}+b^{2}=c_{1}$ and $c$ by $c+b^{2}=c_{2}$. This fixes $g$, and the corresponding admissible vector potentials are now also determined by the relations $(18)$, where $f(t, u, v)$ is an arbitrary function of the indicated variables and $\tilde{a}(t)=-\dot{b}$, $\tilde{b}(t)=\dot{a}$.

It remains to find the form of the admissible scalar potentials for which purpose we will avoid the conditions (10) which are quite complicated to handle. Observe first that $g$ (as in (16) with $\rho=1$ ) has a double eigenvalue $\lambda=c+b^{2}=c_{2}$ and a single eigenvalue $\mu=c-a^{2}-1=c_{2}-c_{1}-1$. From a different perspective, the solution we obtained for admissible vector potentials more or less forces a kind of privileged 
coordinate transformation upon us. Indeed, extending (17), consider the coordinate transformation (similar to the autonomous case in [12])

$$
\begin{aligned}
u & =x_{1}-b(t) x_{3}, \\
v & =x_{2}+a(t) x_{3}, \\
z & =x_{3}+b(t) x_{1}-a(t) x_{2} .
\end{aligned}
$$

The inverse transformation reads

$$
\begin{aligned}
& x_{1}=k^{-1}\left(\left(a^{2}+1\right) u+a b v+b z\right), \\
& x_{2}=k^{-1}\left(a b u+\left(b^{2}+1\right) v-a z\right), \\
& x_{3}=k^{-1}(-b u+a v+z),
\end{aligned}
$$

where $k=a^{2}+b^{2}+1$. If $P(t)$ denotes the coefficient matrix in these inverse relations, it is easy to verify that its first two columns are eigenvectors of $g$ corresponding to the eigenvalue $\lambda$, and the third column is an eigenvector for the eigenvalue $\mu$. Although this $P$ is not an orthogonal matrix, it is still true that $P^{-1} g P$ diagonalizes $g$. Therefore, Proposition 2 guarantees that the equations of motion will decouple into a 2-dimensional system for $u$ and $v$ and a separate second-order equation for $z$. Reversing the argument, this implies that admissible scalar potentials for our original system necessarily must be of the form

$$
V(t, x)=U(t, u, v)+Z(t, z),
$$

$U$ and $Z$ being arbitrary functions of the indicated variables. This concludes the analysis for the case that $\Gamma_{2}^{1}$ in the relations (15) was required to depend explicitly on at least one of the $x$-variables.

What about the case that $\Gamma_{2}^{1}$ depends on time only, say $\Gamma_{2}^{1}=\sigma(t)$ ? Using the representation (15), the matrix of connection coefficients then has the form

$$
\left(\Gamma_{b}^{a}\right)=\left(\begin{array}{ccc}
0 & \sigma & a \sigma+\tilde{a} \\
-\sigma & 0 & b \sigma+\tilde{b} \\
-(a \sigma+\tilde{a}) & -(b \sigma+\tilde{b}) & 0
\end{array}\right) .
$$

As argued before, we can fix the admissible vector potentials, without loss of generality, to be

$$
A^{a}(t, x)=\Gamma_{b}^{a}(t) x^{b}
$$

since the addition of gradient terms can be absorbed in the scalar potential up to a total time-derivative. The curvature condition (9) is automatically satisfied here but, unlike the situation we encountered in our discussion of examples for $n=2$, the remaining conditions (10) do not reduce to equations involving the function $V$ only and in fact turn out to be quite complicated. A different line of approach can be adopted for this situation, which in fact applies to systems with arbitrary dimension and is explained in the next section. 


\section{The case of connection coefficients depending on time only}

The inspiration comes from the discussion on linear systems in [11]: it is a well known practice for the integration of systems of linear second-order equations to eliminate the terms linear in the velocities by an appropriate coordinate transformation. This idea applies just as well for non-linear equations as long as they have linear dependence on the velocities. If the $\Gamma_{b}^{a}$ are functions of time only, the equations we start from, written with matrix notations, are

$$
\ddot{x}+2 \Gamma(t) \dot{x}+\frac{\partial V}{\partial x}(t, x)+\dot{\Gamma}(t) x=0 .
$$

If $U(t)$ is the solution of the matrix differential equation

$$
\dot{U}+\Gamma U=0, \quad U(0)=I_{n},
$$

then $g(t)=\left(U^{-1}\right)^{T} S U^{-1}$ is the solution of the equation $\dot{g}=g \Gamma+(g \Gamma)^{T}$ with initial value $g(0)=S$, and the coordinate transformation $x=U(t) y$ will eliminate the (linear) velocity dependence in the forces. But remember that $\Gamma$ is skew-symmetric in our case, so that (25) implies that $\dot{U}^{T}-U^{T} \Gamma=0$, from which it subsequently follows that $U^{T} \dot{U}+\dot{U}^{T} U=0$. Hence $U^{T} U$ is constant and since $U(0)=I_{n}, U(t)$ will actually be an orthogonal matrix with the identity matrix as initial value. It is further straightforward to verify that the transformed differential equations for the $y$-variables will read:

$$
\ddot{y}-\left(U^{T} \Gamma^{2} U\right) y+\frac{\partial \bar{V}}{\partial y}=0, \quad \text { with } \quad \bar{V}(t, y)=V(t, U(t) y)
$$

or

$$
\ddot{y}+\frac{\partial W}{\partial y}=0, \quad \text { with } \quad W(t, y)=\bar{V}-\frac{1}{2} y^{T}\left(U^{T} \Gamma^{2} U\right) y .
$$

Observe that the $y$-equations still have the unit matrix as multiplier since the forces are of potential type. More interestingly is the fact that the alternative multiplier $g(t)$ for the original equations translates to the existence of a constant alternative multiplier $S$ for the $y$-equations. What's more in making this transition we have eliminated the problem that the remaining conditions (10) had the complicating coupling between scalar and vector potential, because they now simply read

$$
S_{a c} \frac{\partial^{2} W}{\partial y^{c} \partial y^{b}}=S_{b c} \frac{\partial^{2} W}{\partial y^{c} \partial y^{a}}
$$

We summarize our results in the following statement.

Proposition 3. Assume that the SODE $\Gamma$ on $\mathbb{R}^{2 n+1}$ admits a coordinate representation for which $\delta_{a b}$ is a multiplier matrix for the inverse problem of Lagrangian mechanics and that the connection coefficients are functions of time only. Then, without loss of 
generality, the vector potential can be taken to be of the form $A^{a}(t, x)=\Gamma_{b}^{a}(t) x^{b}$, and for there to exist an alternative multiplier, it suffices to construct a non-singular, constant symmetric matrix $S$ (not a multiple of the identity) and a function $W(t, y)$, satisfying the requirements (27). If $U(t)$ is the solution of the matrix differential equation (25), the admissible scalar potentials for the given system will be of the form

$$
V(t, x)=W\left(t, U^{T}(t) x\right)+\frac{1}{2} x^{T} \Gamma^{2}(t) x,
$$

and the corresponding alternative multiplier is given by $g(t)=U(t) S U^{T}(t)$.

It should be emphasized that the orthogonal transformation with the matrix $U$ in this picture is different from the one with $P$ used before: $P$ is dictated by the diagonalization of the multiplier $g$, whereas $U$ is dictated by the matrix of connection coefficients $\Gamma(t)$. In other words there is no reason why the constant matrix $S$ in Proposition 3 (which is the initial value of $g$ ) would be diagonal and therefore, the transformed equations (26) are different from the decoupled equations which Proposition 2 guarantees to exist. One can easily combine the results of both propositions: once an admissible system would have been reduced to the form (26) and we accordingly know the initial value $S$ of the alternative multiplier $g$, it suffices to diagonalize $S$ to find a further transformation which will decouple the system. Conversely, starting from a number of decoupled systems, each of the form $\ddot{z}_{(i)}=-\partial W_{(i)} / \partial z$ for some scalar potential $W_{(i)}$, multiply each subsystem with an appropriate constant $\lambda_{i}$ and then apply an arbitrary constant, orthogonal transformation to the direct sum total system; the result will be a potential system which has the property (27), with $W=\sum \lambda_{i} W_{(i)}$, expressed in the new $y$-variables. Next, select an arbitrary orthogonal matrix $U(t)$ which has the unit matrix as initial value and define $\Gamma(t)$ by $\Gamma:=-\dot{U} U^{T}$. Then, appealing to the results of Proposition 3, equations of motion of electromagnetic type with the desired property should be of the form:

$$
\ddot{x}^{a}=-2 \Gamma_{c}^{a}(t) \dot{x}^{c}-\frac{\partial V}{\partial x^{a}}-\frac{\partial A^{a}}{\partial t},
$$

with

$$
A^{a}=\Gamma_{b}^{a}(t) x^{b}, \quad \text { and } \quad V=W\left(t, U^{T}(t) x\right)+\frac{1}{2} x^{T} \Gamma^{2}(t) x .
$$

We illustrate this procedure by constructing a specific example for $n=3$ by starting somehow in the middle of the converse procedure we just explained, i.e. in the representation (26). Consider the function

$$
W(t, y)=a(t)\left(y_{1}-p y_{2}\right)^{3}, \quad p \text { constant. }
$$

The general symmetric matrix $S$ for which (27) holds with this $W$, is given by

$$
S=\left(\begin{array}{ccc}
s_{1} & k & m p \\
k & s_{1}+(k / p)\left(1-p^{2}\right) & m \\
m p & m & s_{3}
\end{array}\right)
$$


but to avoid expression swell, let us make the following choice for the five free constant parameters still at our disposal: $s_{1}=s_{3}=k=p=1, m=0$. Obviously the $y$-equations we are taking as starting point happen to exhibit a certain feature of decoupling already, they read

$$
\ddot{y}_{1}=-3 a(t)\left(y_{1}-y_{2}\right)^{2}, \quad \ddot{y}_{2}=3 a(t)\left(y_{1}-y_{2}\right)^{2}, \quad \ddot{y}_{3}=0 .
$$

We choose $U(t)$ to represent a simple rotation around de $y_{1}$ axis:

$$
U(t)=\left(\begin{array}{ccc}
1 & 0 & 0 \\
0 & \cos \theta(t) & -\sin \theta(t) \\
0 & \sin \theta(t) & \cos \theta(t)
\end{array}\right)
$$

then $\Gamma:=-\dot{U} U^{T}$ becomes

$$
\Gamma=\left(\begin{array}{ccc}
0 & 0 & 0 \\
0 & 0 & \sigma \\
0 & -\sigma & 0
\end{array}\right)
$$

where we have put $\sigma(t)=\dot{\theta}(t)$. All we need now to write down admissible $x$-equations of the form (29) is the scalar potential $V(t, x)$ : it is given by

$$
V(t, x)=a(t)\left(x_{1}-(\cos \theta) x_{2}-(\sin \theta) x_{3}\right)^{3}-\frac{1}{2} \sigma^{2}\left(x_{2}^{2}+x_{3}^{2}\right) .
$$

It is clear even without writing them down that the $x$-equations will be fully coupled. However, they have the alternative multiplier

$$
g=\left(\begin{array}{ccc}
1 & \cos \theta & \sin \theta \\
\cos \theta & 1 & 0 \\
\sin \theta & 0 & 1
\end{array}\right) .
$$

The matrix $g$ (just like $S$ of course) has three distinct eigenvalues, namely $2,0,1$. Hence, Proposition 2 proclaims that the $x$-equations should decouple completely in appropriate coordinates (and of course the partially coupled $y$-equations we started from just as well). It is a fairly straightforward exercise (at least with some computer algebra assistance) to compute a matrix $P(t)$ which diagonalizes $g(t)$ and to verify that the resulting transformation $x=P(t) z$ does indeed have the predicted decoupling effect. But note with regard to the $y$-equations that the transition to the decoupling $z$-coordinates may have re-introduced velocity terms, resulting from the fact that the matrix $P(t)$ which diagonalizes $g$ need not be taken strictly orthogonal, and that $P^{-1} U$ accordingly need not be constant. Of course, if we diagonalize the constant multiplier $S$ of the $y$-equations via a constant matrix, we obtain another transition to a decoupled $z$-system without velocity terms.

\section{Acknowledgements}

This work is part of the IRSES project GEOMECH (nr. 246981) within the 7th European Community Framework Programme. TM is a Postdoctoral Fellow of the Research Foundation Flanders (FWO). OK acknowledges support by the grant GACR 201/09/0981 of 
the Czech Science Foundation. Each author warmly thanks the others for the hospitality of their institutions while this paper was being written.

\section{References}

[1] J. E. Aldridge, G. E. Prince, W. Sarlet and G. Thompson, An EDS approach to the inverse problem in the calculus of variations, J. Math. Phys. 47 (2006) 103508.

[2] I. Anderson and G. Thompson, The inverse problem of the calculus of variations for ordinary differential equations, Mem. Amer. Math. Soc. 473 (1992).

[3] I. Bucataru and M.F. Dahl, Semi-basic 1-forms and Helmholtz conditions for the inverse problem of the calculus of variations, J. Geom. Mech. 1 (2009) 159-180.

[4] I. Bucataru and O. Constantinescu, Helmholtz conditions and symmetries for the time dependent case of the inverse problem of the calculus of variations, J. Geom. Phys. 60 (2010) 1710-1725.

[5] C. Ferrario, G. Lo Vecchio, G. Marmo, G. Morandi and C. Rubano, A separability theorem for dynamical systems admitting alternative Lagrangian descriptions, J. Phys. A: Math. Gen., 20 (1987) 3225-3236.

[6] J. Grifone and Z. Muzsnay, On the inverse problem of the variational calculus: existence of Lagrangians associated with a spray in the isotropic case, Ann. Inst. Fourier 49 (1999) 1387-1421.

[7] O. Krupková, Variational metrics on $\mathbb{R} \times T M$ and the geometry of nonconservative mechanics, Math. Slovaca 44 (1994) 315-335.

[8] O. Krupková and G. E. Prince, Second order ordinary differential equations in jet bundles and the inverse problem of the calculus of variations, in Handbook of Global Analysis D. Krupka and D. Saunders eds. (Elsevier 2008) 837-904.

[9] E. Massa and E. Pagani, Jet bundle geometry, dynamical connections, and the inverse problem of Lagrangian mechanics, Ann. Inst. H. Poincar, Phys. Thor. 61 (1994) 17-62.

[10] W. Sarlet, The Helmholtz conditions revisited. A new approach to the inverse problem of Lagrangian dynamics, J. Phys. A: Math. Gen. 15 (1982) 1503-1517.

[11] W. Sarlet, E. Engels and L.Y. Bahar, Time-dependent linear systems derivable from a variational principle, Int. J. Engng. Sci. 20 (1982) 55-66.

[12] W. Sarlet and G. Prince, Alternative kinetic energy metrics for Lagrangian systems, J. Phys. A: Math. Theor. 43 (2010) 445204. 
[13] W. Sarlet, A. Vandecasteele, F. Cantrijn and E. Martínez, Derivations of forms along a map: the framework for time-dependent second-order equations, Diff. Geom. Applic. 5 (1995) 171-203. 